\title{
Association between phosphodiesterase type 5 inhibitors use and risk of melanoma: a meta-analysis
}

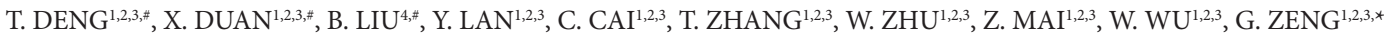 \\ ${ }^{1}$ Department of Urology, Minimally Invasive Surgery Center, The First Affiliated Hospital of Guangzhou Medical University, Guangzhou, China; \\ ${ }^{2}$ Guangzhou Institute of Urology, Guangzhou, China; ${ }^{3}$ Guangdong Key Laboratory of Urology, Guangzhou, China; ${ }^{4}$ The First Affiliated Hospital \\ of Jinan University, Guangzhou, China
}

*Correspondence: gzgyzgh@vip.tom.com

${ }^{*}$ Contribute equally to this work.

Received January 11, 2017/ Accepted June 14, 2017

\begin{abstract}
This meta-analysis aimed to clarify the actual association between the phosphodiesterase type 5 inhibitors (PDE5-Is) use and the risk of melanoma in erectile dysfunction (ED) patients. A systematic literature search was conducted in online databases in October, 2016 to identify studies focusing on the association between PDE5-Is use and the risk of melanoma. Summarized multivariate adjusted risk ratios (RRs) and 95\% confidence intervals (CIs) were calculated to assess the strength of associations. A total of six clinical trials containing more than one million participants were included. ED patients using PDE5-Is shared a significant high risk of melanoma $(\mathrm{RR}=1.12,95 \% \mathrm{CI}=1.03-1.21, \mathrm{p}=0.006)$. Positive associations were observed in all kinds of prescriptions: single prescription ( $R R=1.20,95 \% \mathrm{CI}=1.06-1.35, \mathrm{p}=0.003)$, medium number of prescription $(\mathrm{RR}=1.15,95 \% \mathrm{CI}=1.01-1.30, \mathrm{p}=0.03)$, and high number of prescription $(\mathrm{RR}=1.18,95 \% \mathrm{CI}=1.05-1.34$, $\mathrm{P}=0.006$ ). Additionally, PDE5-Is were also found to be significantly associated with increased risk of basal cell carcinoma $(\mathrm{RR}=1.14,95 \% \mathrm{CI}=1.09-1.19, \mathrm{p}<0.00001)$. Our study indicates that PDE5-Is use could significantly increase the risk of melanoma and basal cell carcinoma. However, the risk of melanoma did not rise significantly with the increased number of prescriptions. Consequently, owing to the lack of information about other potential synergistic factors, it is difficult for us to make a solid conclusion that application of PDE5-Is is the direct cause of increased risk of melanoma. Their relationship needs to be validated by further evidences.
\end{abstract}

Key words: association, melanoma, meta-analysis, phosphodiesterase type 5 inhibitors

Erectile dysfunction (ED), defined as the inability to attain or maintain the penile erection condition adequate for successful vaginal intercourse [1], is a common andrologic disorder with the prevalence rate of $1-10 \%$ among men elder than 40 years old [2]. Although age plays the most important role in the cause of $\mathrm{ED}$, some lifestyle factors also contribute to the ED's onset such as smoking, alcohol misuse, obesity, physical inactivity and sleeping disorders [3]. It is noteworthy that many systematic diseases are also associated with ED closely [4], in which diabetes mellitus has been proved to be a vital risk factor [5]. Since ED not only influences patients' quality of life and their relationship with partners, but also indicates potential combined mental or organic diseases. Therefore, interventions and treatments should be applied on ED patients regularly to prevent further impairments.
Phosphodiesterase type 5 inhibitors (PDE5-Is) remain the first-line treatment of ED, blocking PDE5 to degrade the cyclic GMP (cGMP), which could mediate the release of nitric oxide (NO) to maintain the relaxation of cavernosal smooth muscle contraction [6]. PDE5-Is are considered to have some mild adverse-effects including transient headache, dizziness and blurred vision. Rare but urgent complications also occur such as hypotension and glaucoma [7]. Recently, several studies have raised controversial opinions about whether the use of PDE5-Is is associated with an increased risk of melanoma. The mechanism of melanoma includes a complex process with the activated RAS/RAF/MEK/ ERK pathway promoting the proliferation and survival of melanoma cells, mediated by BRAF or NRAS somatic mutations. PED5A is down-regulated by BRAF in the occurrence of melanoma; hence, the application of PDE5-Is could 
partially mimic the function of BRAF [8-10]. Two kinds of PDE5-Is were also discovered to increase melanin synthesis and accelerate the development of melanoma [11]. Up to now, a total of six clinical trials in five studies [12-16] have been focusing on the association between PDE5-Is and risk of melanoma. Different results were obtained from these studies: two studies reflected a significant association [12, 14]; one found positive relation only in patients receiving single prescription [15], while others revealed the increased risk in patients taking multiple prescriptions of PDE5-Is [13, 16]. Consequently, a combined comprehensive analysis of these relevant studies is necessary to verify the actual influences of PDE5-Is on the risk of melanoma. To our knowledge, it is the first meta-analysis regarding the association between PDE5-Is use and the risk of melanoma. Since melanoma is a life-threatening malignant tumor with poor prognosis, this adverse event caused by PDE5-Is needs more attention by clinicians and our study could provide some evidence and guides for treatments of ED.

\section{Materials and methods}

Search strategy. A systematic literature search was conducted using online databases including Pubmed, Embase, Medline, Cochrane Library, CBM, CNKI and VIP databases in October, 2016. Following search terms were used: "'phosphodiesterase type 5 inhibitors' or 'PDE5-Is' or 'PDE5A inhibitors' or 'PDE5 inhibitors' or 'PDEIs"' and "melanoma". No language restriction was applied. Reference lists of relevant studies were also checked.

Inclusion and exclusion criteria. Studies researching the association between PDE5-Is use and the risk of melanoma in ED patients were included. Accordingly, conference proceedings, editorials, reviews, animal experiments and repeated publications were excluded from this review. Two reviewers (T.D. and X.D.) independently assessed relevant records and disagreement was resolved via open discussion.

Data extraction. Data of eligible studies was examined and collected independently by two reviewers. Following information was extracted using a standard form: authors of study, year of publication, country/region, ethnicity, institution, study period and databases used, type of PDE5-Is, case and control sample size, number of melanoma patients, and relevant outcomes reflecting the association. The level of evidence (LOE) of each eligible study was assessed according to GRADE approach by two reviewers independently [17].

Statistics analysis. Multivariate adjusted hazard ratios (HRs) or odds ratios (ORs) of PDE5-Is for the risk of melanoma and their 95\% confidence intervals (CIs) were extracted from all included studies. Summarized multivariate adjusted risk ratios (RRs) and 95\% CIs were calculated to assess the strength of association between PDE5-Is and melanoma. Subgroup analyses were conducted on different number of prescriptions. In addition, available multivariate adjusted HRs or ORs and 95\% CIs of PDE5-Is use for the risk of basal cell carcinoma (BCC) and squamous cell carcinoma (SCC) were also extracted and pooled. Chi-square test was performed to assess the statistical heterogeneity among studies [18]. If no heterogeneity existed with a p-value $>0.10$, fixed-effect model was used to calculate the combined RR. Otherwise, the random-effect model was applied. Pooled RR could be considered significant only with a two-sided $\mathrm{p}$-value $<0.05$. The effect of publication bias was evaluated by inverted funnel plot visual inspection. All statistical analyses were conducted by RevMan (version 5.3; Cochrane Collaboration, Oxford, UK) and STATA (version 13.0; StataCorp, College Station, Texas, USA) software.

\section{Results}

Eligible studies and characteristics. Figure 1 shows a detailed flow diagram of eligible studies identified for this systematic review. Finally, six clinical trials in five studies [12-16] containing more than one million participants were included, with three prospective cohort studies [12-14] and three retrospective case-control studies [15, 16]. All studies were conducted among European or US populations. The research period ranged from 6 to 17 years, and all studies were published after 2014. In three prospective cohort studies, average melanoma incidence in ED patients using PDE5-Is was 0.32\% (range 0.22-1.02\%); while in participants without PDE5-Is use, average incidence was $0.18 \%$ (range $0.13-0.52 \%)$. Other baseline characteristics of all included studies are shown in Table 1.

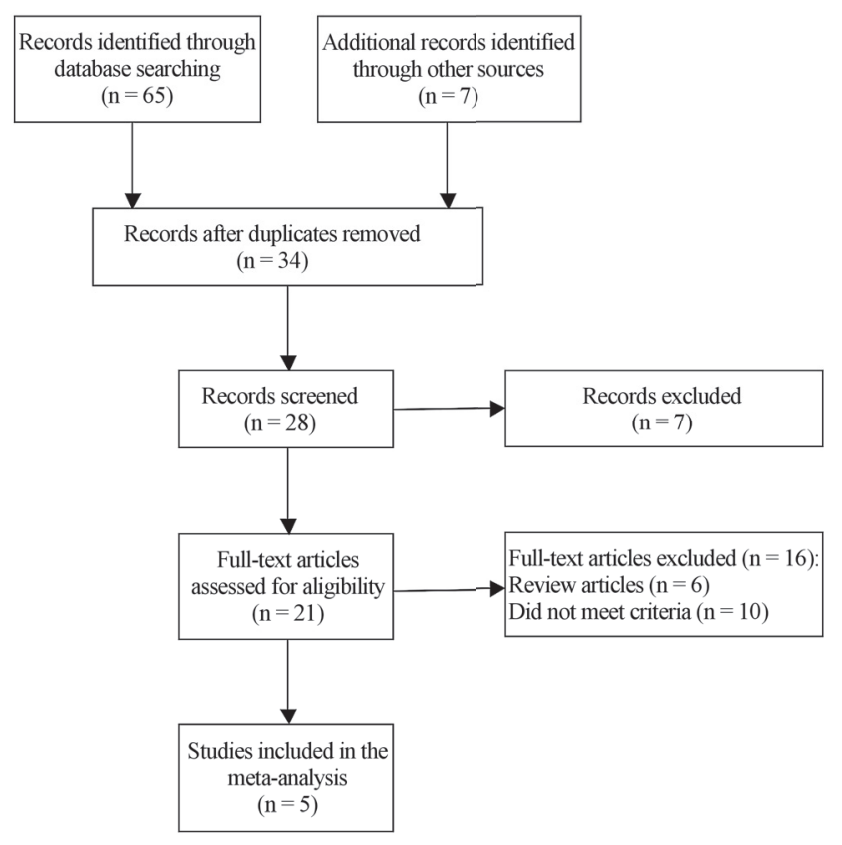

Figure 1. Flow diagram of the meta-analysis. 
Association between PDE5-Is use and risk of melanoma. Multivariate adjusted HRs or ORs with 95\% CIs of PDE5-Is for the risk of melanoma were extracted from all five studies. Our metaanalysis indicates that ED patients using PDE5-Is shared a significant high risk of melanoma $(\mathrm{RR}=1.12,95 \% \mathrm{CI}=1.03-1.21, \mathrm{p}=0.006)$ with slight heterogeneity among five studies $\left(\mathrm{I}^{2}=48 \%, \mathrm{p}=0.09\right)$ (Figure 2). Additionally, subgroup analysis was conducted based on the number of prescriptions (three studies [13-15] were included). As a result, positive associations were observed in all kinds of prescriptions (single prescription: $\mathrm{RR}=1.20$, 95\% $\mathrm{CI}=1.06-1.35, \mathrm{p}=0.003$; medium number of prescriptions: $\mathrm{RR}=1.15,95 \% \mathrm{CI}=1.01-1.30$, $\mathrm{p}=0.03$; high number of prescriptions: $\mathrm{RR}=1.18$, $95 \% \mathrm{CI}=1.05-1.34, \mathrm{p}=0.006$ ) (Figure 3). No publication bias was detected among five included studies through inverted funnel plot.

Association between PDE5-Is use and risk of non-melanoma skin cancers. Four studies [12-15] also investigated the association between PDE5-Is use and the risk of BCC. Slight heterogeneity was found among them $\left(\mathrm{I}^{2}=54 \%, \mathrm{p}=0.09\right)$, and the result of our meta-analysis demonstrated that PDE5-Is use was significantly associated with increased risk of $\mathrm{BCC}(\mathrm{RR}=1.14,95 \% \mathrm{CI}=1.09$ $1.19, \mathrm{p}<0.00001$ ) (Figure 4). Moreover, two studies $[12,13]$ concentrated on the influence of PDE5-Is use on the risk of SCC, but no significant difference was observed $(\mathrm{RR}=1.02,95 \% \mathrm{CI}=0.83-1.25$, $\mathrm{p}=0.88)$.

\section{Discussion}

To the best of our knowledge, this is the first meta-analysis aiming to evaluate the association between PDE5-Is use and the risk of melanoma in ED patients. According to the summarized results from cohort studies included in our review, the average melanoma incidence of participants using PDE5-Is or not was $0.32 \%$ and $0.18 \%$, respectively. Our analysis demonstrated that the application of PDE5-Is was significantly associated with increased risk of melanoma and BCC. Furthermore, the association between PDE5-Is use and risk of melanoma always turned out to be positive regardless of the number of PDE5-Is prescriptions. However, the risk of melanoma did not increase with the incremental number of prescriptions.

Potential biological mechanisms of PDE5-Is in the occurrence of melanoma were considered to include several aspects. Classic RAS/RAF pathway played a crucial role in melanoma, which originally arose from melanocytes serving as protection

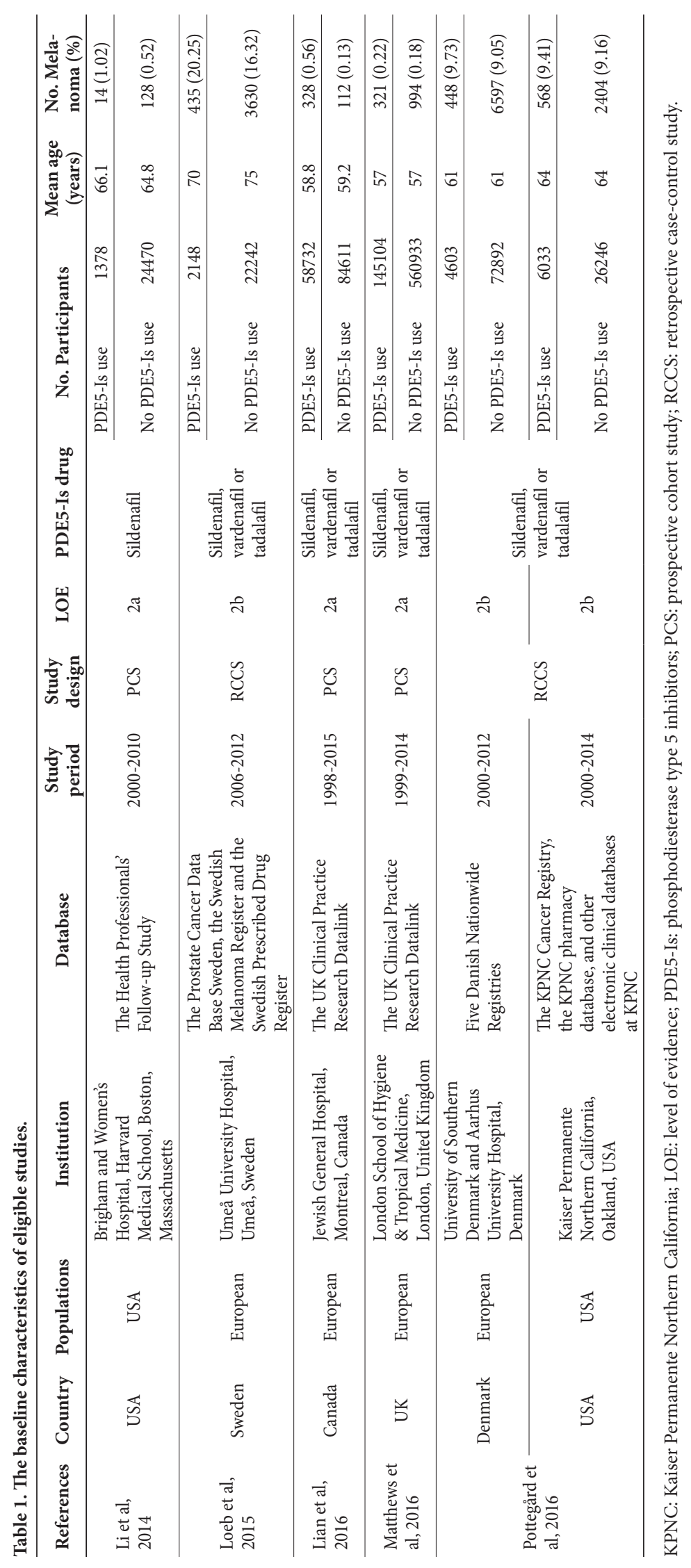




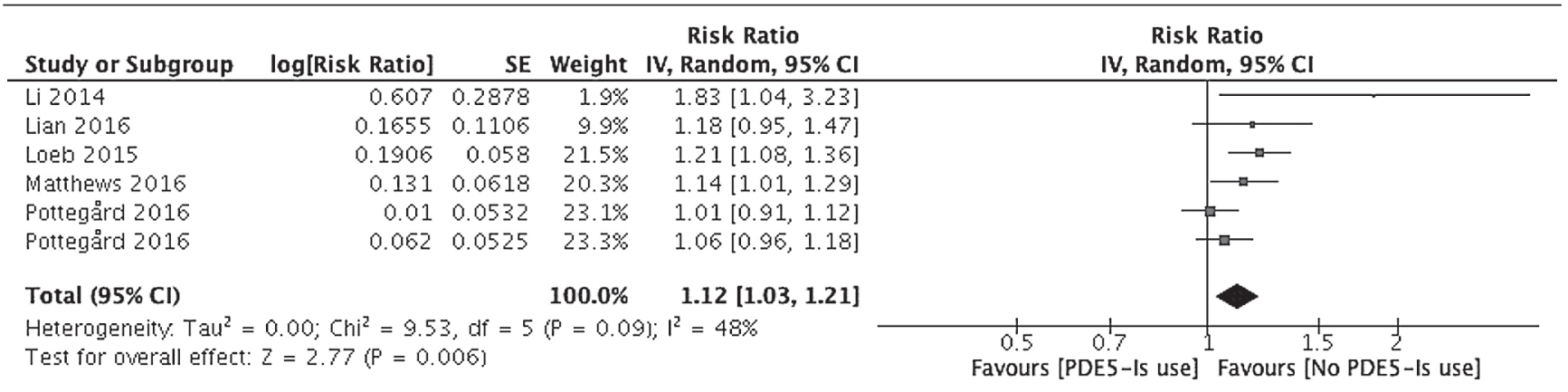

Figure 2. Forest plot for association between PDE5-Is use and risk of melanoma.

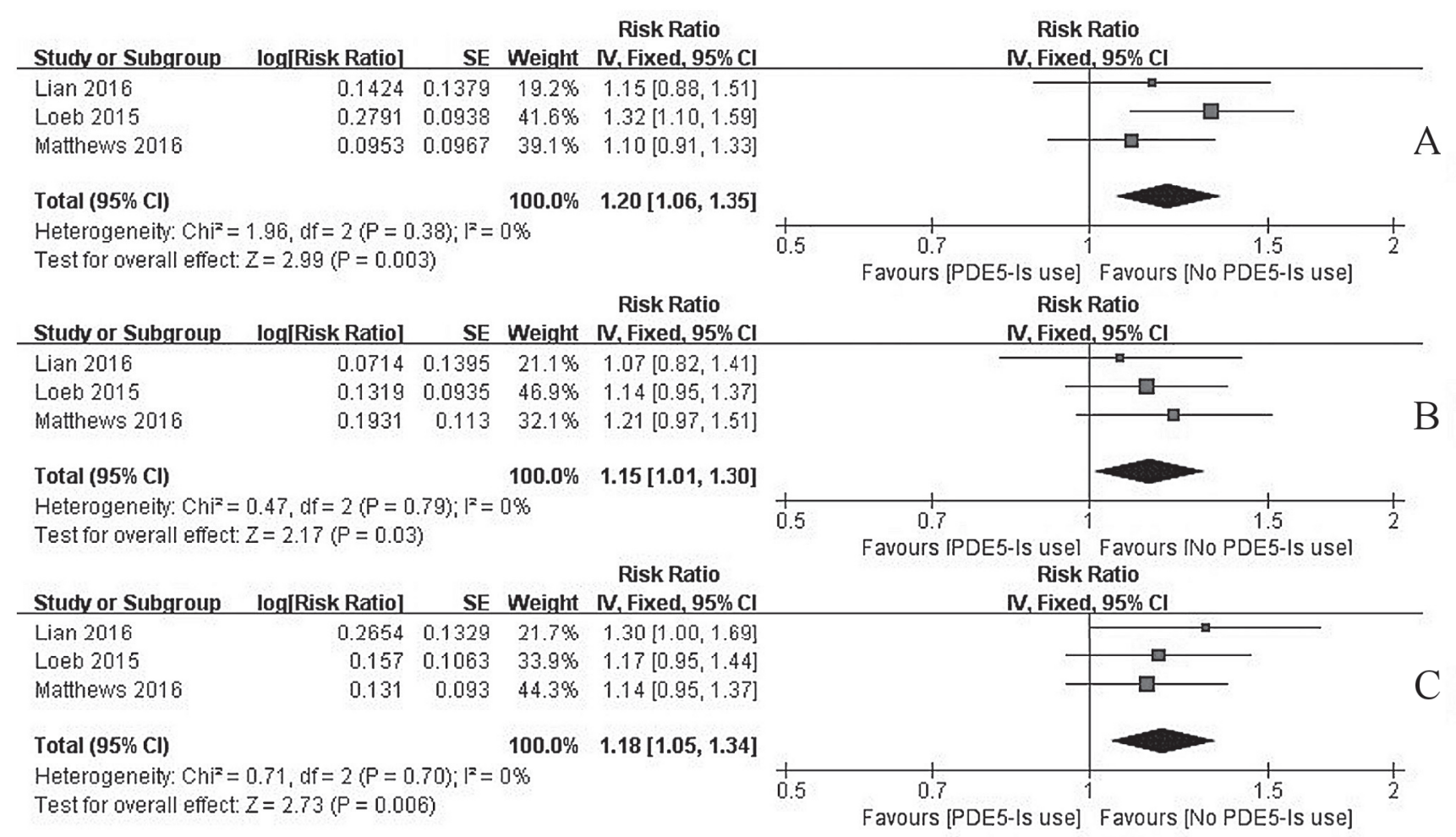

Figure 3. Forest plot for association between different number of PDE5-Is prescriptions and risk of melanoma (A: Single prescription; B: Medium number of prescriptions; C: High number of prescriptions).

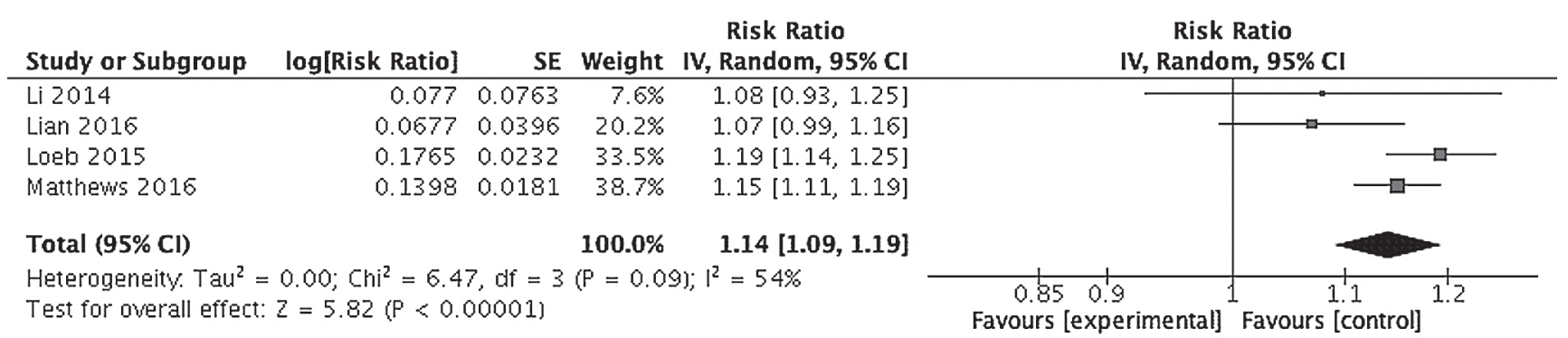

Figure 4. Forest plot for association between PDE5-Is use and risk of basal cell carcinoma.

from ultraviolet radiation [19]. Among three kinds of RAS (HRAS, KRAS, NRAS) and three RAF (ARAF, BRAF, CRAF) genes, NRAS and BRAF were responsible for the majority of oncogenic mutations [20]. The activated BRAF probably resulted in the downregulation of cGMP-dependent PDE5A by increasing the expression of one transcription factor called BRN2 [21], which was proved to cause the carcinoma invasion [22]. BRN2 was observed widely expressed 
in melanoblasts $[23,24]$ and shared a binding site in PDE5A promoter in order to suppress its transcription. Application of PDE5-Is could act as an incentive source like mutated BRAF [25] and it might be more apparent in those cases without BRAF mutations [13]. Besides, MEK/ERK pathway was disturbed by PDE5-Is in a similar way to RAS/RAF, which might not be found in the pathogenesis of melanoma caused by some kinds of PDE5-I, such as sildenafil [26]. Consequently, PDE5-Is increased the concentration of intracellular cGMPs through inflow of $\mathrm{Ca}^{2+}$, suppressing a series of cellular physiological functions and leading to more severe invasion of the cancer [27-29]. Former studies also discovered the interaction between RAS/RAF way and cAMP signaling in melanoma cells [30].

Our analysis indeed proved a significant association between any prescriptions of PDE5-Is use and melanoma. Nevertheless, the dose-response relationship was not observed between PDE5-Is use and risk of melanoma, and PDE5-Is use was also validated to be associated with BCC, in which PDE5 pathway was not considered to be involved. Other factors that could be synergic with the use of PDE5-Is were researched at the same time [31, 32]. People with a higher socioeconomic status tend to be more accessible to PDE5-Is and have been confirmed to be more susceptible to malignant melanoma [33]. Confounding related lifestyle factors could also contribute to some degree, such as tanning bed usage, recreational ultraviolet exposure and obesity [34, 35]. On the contrary, better education and income leads to more frequent uses of sunscreens and other sun-protective instruments [36, 37]. Therefore, relationship between PDE5-Is and melanoma is still indefinite and needs to be validated by further evidence.

Several potential limitations of this analysis should be addressed. First, all eligible studies of this meta-analysis were cohort studies or case-control studies, and no randomized controlled trials (RCT) were included, which might potentially influence the strength of associations in our study. However, no RCTs related to association between PDE5-Is use and the risk of melanoma exist yet, and it also seems impossible to conduct such a RCT on this topic. Second, data of some studies were incomplete even by contacting the authors. Because the exact classification of prescription number in each study could not be completely the same, we had to classify them into groups of single, medium and high number of prescriptions. Finally, all included studies in our analysis were conducted in European or US populations, and that may potentially bring some limitations for applying our conclusions for other races.

\section{Conclusion}

Our study found that PDE5-Is use in ED patients was significantly associated with increased risk of melanoma and basal cell carcinoma. In addition, regardless of the number of prescriptions, the association between PDE5-Is use and the risk of melanoma demonstrated as positive results. However, the risk of melanoma did not rise with the increased number of prescriptions. Therefore, owing to not only the lack of this significance but also lacking information about other potential synergistic factors, it is difficult for us to make a solid conclusion that application of PDE5-Is could cause the increased risk of melanoma directly.

\section{References}

[1] SHAMLOUL R, GHANEM H. Erectile dysfunction. Lancet 2013; 381: 153-165. doi: 10.1016/S0140-6736(12)60520-0

[2] LEWIS RW, FUGL-MEYER KS, CORONA G, HAYES RD, LAUMANN EO et al. Definitions/epidemiology/risk factors for sexual dysfunction. J Sex Med 2010; 7: 1598-1607. doi: 10.1111/j.1743-6109.2010.01778.x

[3] GATTI A, MANDOSI E, FALLARINO M, RADICIONI A, MORINI E et al. Metabolic syndrome and erectile dysfunction among obese non-diabetic subjects. J Endocrinol Invest 2009; 32: 542-545. doi: 10.1007/BF03346504

[4] KÖSEOĞLU N, KÖSEOĞLU H, CEYLAN E, CIMRIN HA, OZALEVLI $S$ et al. Erectile dysfunction prevalence and sexual function status in patients with chronic obstructive pulmonary disease. J Urol 2005; 174: 249-252. doi: 10.1097/01. ju.0000163259.33846.74

[5] ZIMMET PA, ALBERT KG, SHAW J. Global and societal implications of the diabetes epidemic. Nature 2001; 414: 782-787. doi: 10.1038/414782a

[6] LUE TF. Erectile dysfunction. N Engl J Med 2000; 342: 1802 1813. doi: 10.1056/NEJM200006153422407

[7] HAWKSWORTH DJ, BURNETT AL. Pharmacotherapeutic management of erectile dysfunction. Clin Pharmacol Ther 2015; 98: 602-610. doi: 10.1002/cpt.261

[8] HAUSCHILD A, GROB JJ, DEMIDOV LV, JOUARY T, GUTZMER R et al. Dabrafenib in SR/IF-mutated metastatic melanoma: a multicentre, open-label, phase 3 randomised controlled trial. Lancet 2012; 380: 358-365. doi: 10.1016/ S0140-6736(12)60868-X

[9] DAVIES H, BIGNELL GR, COX C, STEPHENS P, EDKINS $S$ et al. Mutations of the BRAF gene in human cancer. Nature 2002; 417: 949-954. doi: 10.1038/nature00766

[10] BOLLAG G, TSAI J, ZHANG J, ZHANG C, IBRAHIM P et al. Vemurafenib: the first drug approved for BRAF-mutant cancer. Nat Rev Drug Discov 2012; 11: 873-886. doi: $10.1038 / \mathrm{nrd} 3847$

[11] NOONAN FP, ZAIDI MR, WOLNICKA-GLUBISZ A, ANVER MR, BAHN J et al. Melanoma induction by ultraviolet A but not ultraviolet $\mathrm{B}$ radiation requires melanin pigment. Nat Commun 2012; 3: 884. doi: 10.1038/ncomms1893

[12] LI WQ, QURESHI AA, ROBINSON KC, HAN J. Sildenafil use and increased risk of incident melanoma in US men: a prospective cohort study. JAMA Intern Med 2014; 174: $964-$ 970. doi: 10.1001/jamainternmed.2014.594

[13] LIAN Y, YIN H, POLLAK MN, CARRIER S, PLATT RW et al. Phosphodiesterase Type 5 Inhibitors and the Risk of Melanoma Skin Cancer. Eur Urol 2016; 70: 808-815. doi: 10.1016/j.eururo.2016.04.035 
[14] MATTHEWS A, LANGAN SM, DOUGLAS IJ, SMEETH L, BHASKARAN K. Phosphodiesterase Type 5 Inhibitors and Risk of Malignant Melanoma: Matched Cohort Study Using Primary Care Data from the UK Clinical Practice Research Datalink. PLoS Med 2016; 13: e1002037. doi: 10.1371/journal.pmed.1002037

[15] LOEB S, FOLKVALJON Y, LAMBE M, ROBINSON D, GARMO H et al. Use of Phosphodiesterase Type 5 Inhibitors for Erectile Dysfunction and Risk of Malignant Melanoma. JAMA 2015; 313: 2449-2455. doi: 10.1001/jama.2015.6604

[16] POTTEGÅR A, SCHMIDT SA, OLESEN AB, ACHACOSO N, VAN DEN EEDEN SK et al. Use of sildenafil or other phosphodiesterase inhibitors and risk of melanoma. Br J Cancer 2016; 115: 895-900. doi: 10.1038/bjc.2016.248

[17] GUYATT GH, OXMAN AD, VIST GE, KUNZ R, FALCKYTTER Y et al. GRADE: an emerging consensus on rating quality of evidence and strength of recommendations. BMJ 2008; 336: 924-926. doi: 10.1136/bmj.39489.470347.AD

[18] HIGGINS JP, THOMPSON SG, DEEKS JJ, ALTMAN DG. Measuring inconsistency in meta-analyses. BMJ 2003; 327: 557-560. doi: 10.1136/bmj.327.7414.557

[19] AROZARENA I, SANCHEZ-LAORDEN B, PACKER L, HIDALGO-CARCEDO C, HAYWARD R et al. Oncogenic BRAF induces melanoma cell invasion by downregulating the cGMPspecific phosphodiesterase PDE5A. Cancer Cell 2011; 19: 45-57. doi: 10.1016/j.ccr.2010.10.029

[20] GRAY-SCHOPFER V, WELLBROCK C, MARAIS R. Melanoma biology and new targeted therapy. Nature 2007; 445: 851-857. doi: 10.1038/nature05661

[21] GOODALL J, CARREIRA S, DENAT L, KOBI D, DAVIDSON I et al. Brn-2 represses microphthalmia-associated transcription factor expression and marks a distinct subpopulation of microphthalmia-associated transcription factornegative melanoma cells. Cancer Res 2008; 68: 7788-7794. doi: 10.1158/0008-5472.CAN-08-1053

[22] PINNER S, JORDAN P, SHARROCK K, BAZLEY L, COLLINSON L et al. Intravital imaging reveals transient changes in pigment production and Brn2 expression during metastatic melanoma dissemination. Cancer Res 2009; 69: 79697977. doi: 10.1158/0008-5472.CAN-09-0781

[23] COOK AL, DONATIEN PD, SMITH AG, MURPHY $\mathrm{M}$, JONES MK et al. Human melanoblasts in culture: expression of BRN2 and synergistic regulation by fibroblast growth factor-2, stem cell factor, and endothelin-3. J Invest Dermatol 2003; 121: 1150-1159. doi: 10.1046/j.15231747.2003.12562.x

[24] YAMAGUCHI Y, BRENNER M, HEARING VJ. The regulation of skin pigmentation. J Biol Chem 2007; 282: 2755727561. doi: 10.1074/jbc.R700026200

[25] Omori K, Kotera J. Overview of PDEs and their regulation. Circ Res 2007; 100: 309-327. doi: 10.1161/01. RES.0000256354.95791.f1
[26] PACKER LM, EAST P, REIS-FILHO JS, MARAIS R. Identification of direct transcriptional targets of (V600E)BRAF/ MEK signalling in melanoma. Pigment Cell Melanoma Res 2009; 22: 785-798. doi: 10.1111/j.1755-148X.2009.00618.x

[27] FAJARDO AM, PIAZZA GA, TINSLEY HN. The role of cyclic nucleotide signaling pathways in cancer: targets for prevention and treatment. Cancers (Basel) 2014; 6: 436-458. doi: $10.3390 /$ cancers 6010436

[28] FEIL R, LOHMANN SM, DE JONGE H, WALTER U, HOFMANN F. Cyclic GMP-dependent protein kinases and the cardiovascular system: insights from genetically modified mice. Circ Res 2003; 93: 907-916. doi: 10.1161/01. RES.0000100390.68771.CC

[29] FEIL R, FEIL S, HOFMANN F. A heretical view on the role of $\mathrm{NO}$ and cGMP in vascular proliferative diseases. Trends Mol Med 2005; 11: 71-75. doi: 10.1016/j.molmed.2004.12.001

[30] Dumaz N, Marais R. Integrating signals between cAMP and the RAS/RAF/MEK/ERK signalling pathways. Based on the anniversary prize of the Gesellschaft fur Biochemie und Molekularbiologie Lecture delivered on 5 July 2003 at the Special FEBS Meeting in Brussels. FEBS J 2005; 272: 34913504. doi: 10.1111/j.1742-4658.2005.04763.x

[31] PLYM A, FOLKVALJON Y, GARMO H, HOLMBERG L, JOHANSSON E et al. Drug prescription for erectile dysfunction before and after diagnosis of localized prostate cancer. J Sex Med 2014; 11: 2100-2108. doi: 10.1111/jsm.12586

[32] NORDIN M, DACKEHAG M, GERDTHAM UG. Socioeconomic inequalities in drug utilization for Sweden: evidence from linked survey and register data. Soc Sci Med 2013; 77: 106-117. doi: 10.1016/j.socscimed.2012.11.013

[33] JIANG AJ, RAMBHATLA PV, EIDE MJ. Socioeconomic and lifestyle factors and melanoma: a systematic review. Br J Dermatol 2015; 172: 885-915. doi: 10.1111/bjd.13500

[34] Robsahm TE, Tretli S. Cutaneous malignant melanoma in Norway: variation by region of residence before and after the age 17. Cancer Causes Control 2001; 12: 569-576.

[35] SIMIC D, SITUM M, MARIJANOVIC I, HADZIGRAHIĆ $\mathrm{N}$. Most common skin tumours in correlation with solar ultraviolet radiation in the area of West Herzegovina. Coll Antropol 2011; 35: 1129-1134.

[36] IDORN LW, WULF HC. Socioeconomic status and cutaneous malignant melanoma in Northern Europe. Br J Dermatol 2014; 170: 787-793. doi: 10.1111/bjd.12800

[37] GAVIN A, BOYLE R, DONNELLY D, DONNELLY C, GORDON S et al. Trends in skin cancer knowledge, sun protection practices and behaviours in the Northern Ireland population. Eur J Public Health 2012; 22: 408-412. doi: 10.1093/eurpub/ckr087 